\title{
THE MONODROMY OF CERTAIN FAMILIES OF LINEAR SERIES IS AT LEAST THE ALTERNATING GROUP
}

\author{
DAN EDIDIN
}

(Communicated by Maurice Auslander)

\begin{abstract}
Let $g, r, d$ be positive integers such that the Brill-Noether number, $\rho(g, r, d):=g-(r+1)(g-d+r)=0$. We prove that if $r+1 \neq g-d+r$, then for suitable families of curves $(C / B)$, the monodromy of the family $G_{d}^{r}(C / B) \rightarrow B$ is at least the alternating group. Our techniques are combinatorial, and similar to those used by Bercov and Proctor in their paper [BP].
\end{abstract}

\section{INTRODUCTION}

Let $g, r, d$ be positive integers such that the Brill-Noether number, $\rho(g, r, d):=g-(r+1)(g-d+r)=0$. It is known (see for example [ACGH]), that a general curve of genus $g$ has exactly

$$
N(g, r, d):=g ! \prod_{i=0}^{i=r} \frac{i !}{(g-d+r+i) !}
$$

distinct $g_{d}^{r}$ 's. In their paper [EH], Eisenbud and Harris proved that there is a family of smooth curves $\mathscr{C} / B$ such that the family $G_{d}^{r}(\mathscr{C} / B) \rightarrow B$ has fibers consisting of $N(g, r, d)$ distinct points, and that the monodromy of the family acts transitively on the fibers. If $r=1$ or $g-d+r=2$ (the case of a pencil, and its residual), they showed that the monodromy was in fact $S_{N}$.

The condition on the family $\mathscr{C} / B$ in [EH] is that the family must contain as stable limits all curves of the form shown in Figure 1, for all relative positions of the points $p_{1}, \ldots, p_{g}$.

In this paper we prove the following theorem.

Theorem 1. For a family $\mathscr{C} / B$ satisfying the above condition, the monodromy of $G_{d}^{r}(\mathscr{C} / B) \rightarrow B$ is at least the alternating group as long as $r+1 \neq g-d+r$.

If $r+1=g-d+r$ then a $g_{d}^{r}$ is residual to a $g_{d}^{r}$. In this case, one expects the residual pairing of points in a fiber to be preserved by the monodromy, so the action would not be doubly transitive.

Received by the editors May 25, 1990 and, in revised form, July 25, 1990.

1980 Mathematics Subject Classification (1985 Revision). Primary 14H10. 


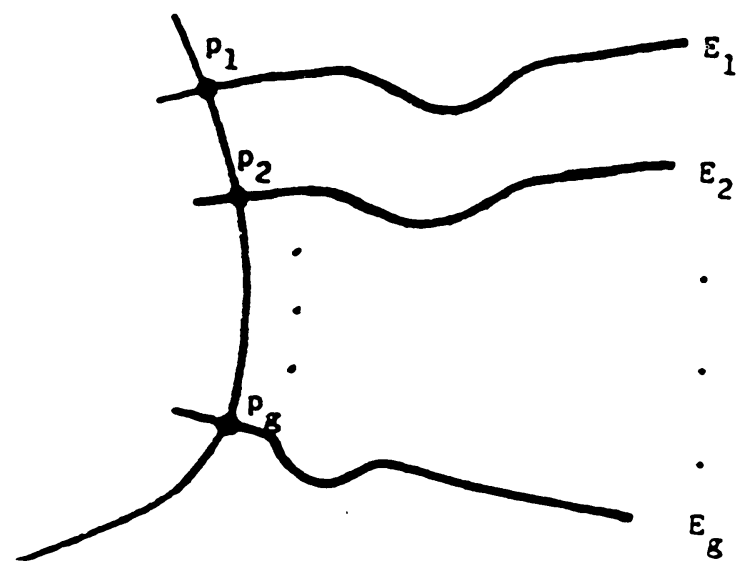

FIGURE 1. $Y$ is rational, $E_{1}, \ldots, E_{g}$ elliptic.

Eisenbud and Harris constructed elements of the monodromy by considering the monodromy along one-dimensional families, $C_{i, p}$, where the values of $p$ are in the projective line. For each pair $(i, p)$ with $p$ finite, the curve has the form.

The curve $C_{i, \infty}=C_{\infty}$ has semistable form shown in Figure 3 (see p. 914).

Eisenbud and Harris showed that the group generated by elements of this form is a transitive subgroup of $S_{N}$ (where $N=N(g, r, d)$ ). To prove Theorem 1 we prove the following combinatorial theorem.

Theorem 2. If $r+1>2$ and $g-d+r>2$ and $g-d+r \neq r+1$, then the group generated by the above elements is either $A_{N}$ or $S_{N}$. If $r+1=g-d+r$, then it is a proper subgroup of $A_{N}$.

A statement of this form was originally given by Bercov and Proctor in their paper [BP]. However, their statement included the rather surprising result that if $r+1=g-d+r$, then the monodromy is also $A_{N}$. The difficulty was resolved when we observed that the theorem [EH, Theorem 3.1] describing the monodromy on families $C_{i, p}$, was incorrectly stated (an $\alpha$ was substituted for an $a$ ). Although the calculations in [BP] of groups generated by elements are correct, these groups are not necessarily subgroups of the monodromy because of the error in the statement of Theorem 3.1 of [EH]. This paper begins with the correct statement of Theorem 3.1 and reperforms the computations of [BP] with the correct monodromy generators.

As was done by Bercov and Proctor [BP], we interpret $g_{d}^{r}$ 's on the curve $C_{\infty}$ as $r+1 \times g-d+r$ Young tableaux. The mondromy group can then be represented as a group action on these tableaux. We show, with an argument specific to our action, that this action is doubly transitive as long as $r+1 \neq g-d+r$ (this is the key lemma in the paper). Furthermore, we can find an element that moves "relatively few" of the tableaux. Using an idea of Bercov and Proctor, we apply 


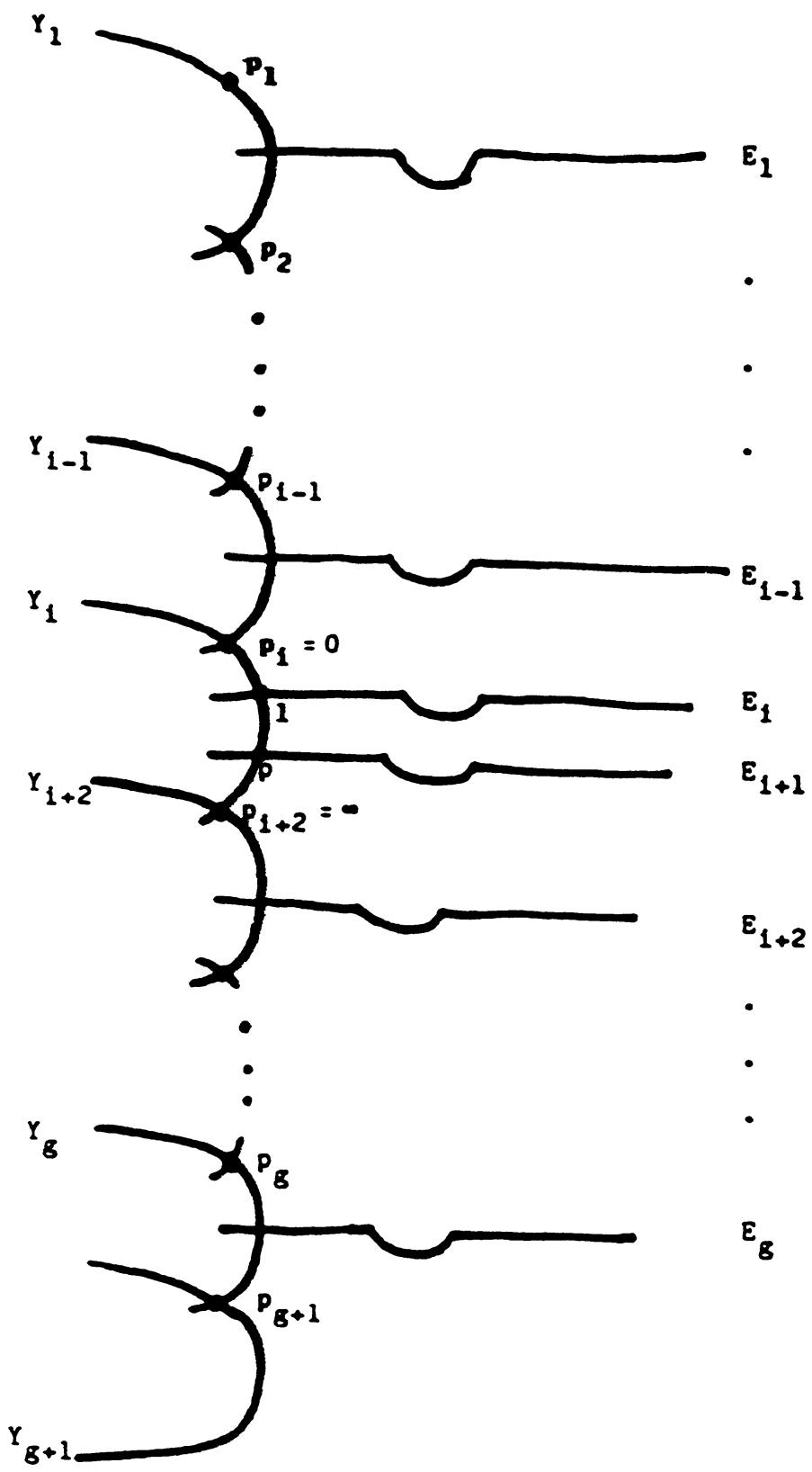

FIGURE 2. $Y_{1}, \ldots, Y_{g+1}$ rational $E_{1}, \ldots, E_{g}$ elliptic.

a theorem of Bochert and can conclude that our group is either $A_{N}$ or $S_{N}$. Lastly, we show, in the case $r+1=g-d+r$, that all of our generators are even. 


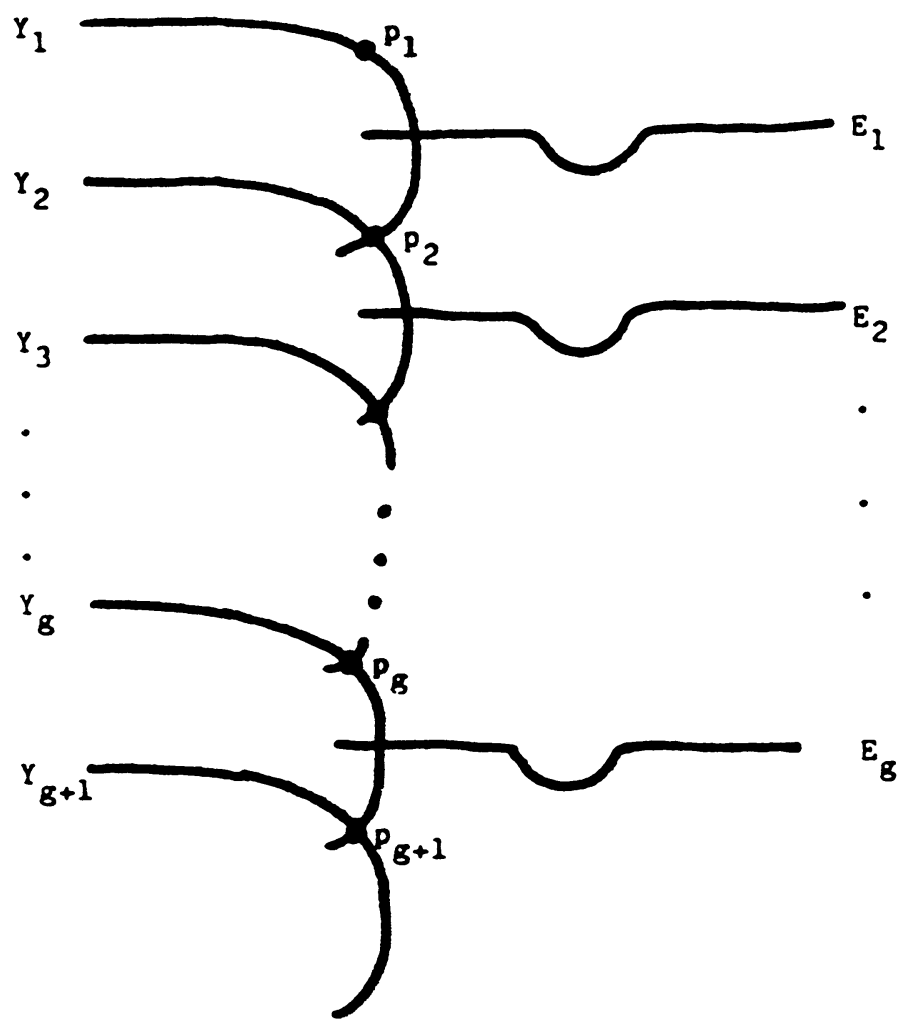

FIGURE 3. $Y_{1}, \ldots, Y_{g+1}$ rational $E_{1}, \ldots, E_{g}$ elliptic.

\section{1. $g_{d}^{r}$ 'S AND Young TABLEAUX}

In this section we explain how $g_{d}^{r}$ 's on $C_{\infty}$ can be identified with $(r+1) \times$ $(g-d+r)$ Young tableaux, and interpret the mondromy action as an action on these Young tableaux. By $\S 2$ of $[\mathbf{E H}], g_{d}^{r}$ 's on a curve $C_{\infty}$ correspond to maximal chains of Schubert cycles $\sigma_{0,0, \ldots, 0} \supset \cdots \supset \sigma_{g-d+r, \ldots, g-d+r}$ with

$$
\alpha_{j}^{(i+1)}=\alpha_{j}^{(i)}
$$

for a unique value of $j$ and

$$
\alpha_{k}^{(i+1)}=\alpha_{k}^{(i)}+1
$$

for $k \neq j$, where $\sigma_{\alpha_{0}^{(i)} \ldots \alpha_{r}^{(i)}}$ is the $i$ th Schubert cycle in the sequence.

Definition 1. An $M \times N$ Young tableau is an $M \times N$ grid $T=T_{i, j}$ containing the numbers $1,2, \ldots, M N$ such that $T_{i, j}<T_{i+1, j}$ and $T_{i, j}<T_{i, j+1}$ for all $i, j$ between 1 and $M \times N$. 
Note. We use standard matrix conventions $-M$ is the number of rows, and $N$ is the number of columns. The entry $T_{i, j}$ refers to the entry in the $i$ th row and $j$ th column.

Given a maximal chain of Schubert cycles, we can associate to it a Young tableau by the following algorithm. At each level $c>0$ in the chain, there is a unique pair $(c, j)$ such that $\alpha_{j}^{(c-1)}=\alpha_{j}^{(c)}$. Enter the value $c$ in the first unfilled entry in the $j$ th column. In terms of notation,

$$
T_{i, j+1}=c \quad \text { if and only if } \alpha_{j}^{(c-1)}=\alpha_{j}^{(c)}=c-i .
$$

(We shift the indexing by one because we start indexing at 0 in the ramification sequences, whereas we start indexing at $(1,1)$ in the tableau.) To see that this procedure produces a Young tableau, we must show that the numbers increase along both rows and columns. By the above description, it is clear that the numbers increase going down columns. Also if $c=T_{i, j} \geq c^{\prime}=T_{i, j+1}$, then we would have $\alpha_{j-1}^{(c-1)} \geq \alpha_{j}^{\left(c^{\prime}\right)}$. This, however, is impossible since

$$
\alpha_{j}^{\left(c^{\prime}\right)} \geq \alpha_{j}^{(c)}=\alpha_{j}^{(c-1)}+1>\alpha_{j-1}^{(c-1)}
$$

Likewise, given an $(r+1) \times(g-d+r)$ Young tableau, we can construct a maximal chain of Schubert cycles by reversing the above procedure. The following example is illustrative.

Example 1. We consider $g_{4}^{1}$ 's on a curve of genus 6. In this case $g=6, r=1$, $d=4$, so we are looking at $2 \times 3$ Young tableaux. $N(g, r, d)=5$ so there are $5 g_{4}^{1}$ 's. The chains of Schubert cycles and their corresponding Young tableaux are enumerated as follows.

$$
\begin{aligned}
& (0,0) \rightarrow(0,1) \rightarrow(1,1) \rightarrow(1,2) \rightarrow(2,2) \rightarrow(2,3) \rightarrow(3,3) \begin{array}{ll}
1 & 2 \\
3 & 4 \\
5 & 6
\end{array} \\
& (0,0) \rightarrow(0,1) \rightarrow(1,1) \rightarrow(1,2) \rightarrow(1,3) \rightarrow(2,3) \rightarrow(3,3) \begin{array}{ll}
1 & 2 \\
3 & 5 \\
4 & 6 \\
\hline
\end{array} \\
& (0,0) \rightarrow(0,1) \rightarrow(0,2) \rightarrow(1,2) \rightarrow(2,2) \rightarrow(2,3) \rightarrow(3,3) \\
& (0,0) \rightarrow(0,1) \rightarrow(0,2) \rightarrow(1,2) \rightarrow(1,3) \rightarrow(2,3) \rightarrow(3,3) \begin{array}{ll}
1 & 3 \\
2 & 4 \\
5 & 6 \\
\hline 1 & 3 \\
2 & 5 \\
4 & 6 \\
\hline 1 & 4 \\
2 & 5 \\
3 & 6
\end{array}
\end{aligned}
$$

We translate the monodromy action described in terms of chains of Schubert 
cycles, to an action on Young tableaux. First let us recall the (correct) action as described in [EH, Theorem 3.1]. For each pair of positive integers $c$ and $a$, we construct a permutation $\pi_{(c, a)}$, which is the product of all transpositions of pairs of chains

and

$$
\sigma_{0,0 \ldots, 0} \supset \cdots \supset \sigma_{\underline{\alpha}^{(c-1)}} \supset \sigma_{\underline{\alpha}^{(c)}} \supset \sigma_{\underline{\alpha}^{(c+1)}} \cdots
$$

$$
\sigma_{0,0 \ldots, 0} \supset \cdots \supset \sigma_{\underline{\beta}^{(c-1)}} \supset \sigma_{\underline{\beta}^{(c)}} \supset \sigma_{\underline{\beta}^{(c+1)}} \cdots
$$

such that

$$
\begin{gathered}
\underline{\alpha}^{(0)}=\underline{\beta}^{(0)}, \ldots, \underline{\alpha}^{(c-1)}=\underline{\beta}^{(c-1)}, \underline{\alpha}^{(c+1)}=\underline{\beta}^{(c+1)} \cdots \\
\alpha_{i}^{(c)}=\beta_{i}^{(c)} \quad \text { for } i \neq j, k \text { and } a:=a_{j}^{(c-1)}-a_{k}^{(c-1)}(j>k \text { by assumption }),
\end{gathered}
$$

where $\underline{a}^{(c)}$ is the vanishing sequence associated to the ramification sequence $\underline{\alpha}^{(c)}$, i.e. $a_{k}^{(c)}=\alpha_{k}^{(c)}+k$.

(Note. In the original statement of [EH, Theorem 3.1], the value of $a$ was defined to be $a=\alpha_{j}^{(c-1)}-\alpha_{k}^{(c-1)}$, i.e. the difference in ramification indices, rather than vanishing indices.)

Let us see how this action works in an example.

Example 2. Again, we consider $g_{4}^{1}$ 's on a curve of genus 6. Consider elements of the form $\pi_{2, a}$. An element $\pi_{2, a}$ acts on pairs of chains that differ only in the 2 nd level. In particular, it possibly exchanges the following pairs of chains.

$$
(0,0) \rightarrow(0,1) \rightarrow(1,1) \rightarrow(1,2) \rightarrow(2,2) \rightarrow(2,3) \rightarrow(3,3)
$$

and

$$
\begin{aligned}
& (0,0) \rightarrow(0,1) \rightarrow(0,2) \rightarrow(1,2) \rightarrow(2,2) \rightarrow(2,3) \rightarrow(3,3) \\
& (0,0) \rightarrow(0,1) \rightarrow(1,1) \rightarrow(1,2) \rightarrow(1,3) \rightarrow(2,3) \rightarrow(3,3)
\end{aligned}
$$

and

$$
(0,0) \rightarrow(0,1) \rightarrow(0,2) \rightarrow(1,2) \rightarrow(1,3) \rightarrow(2,3) \rightarrow(3,3) .
$$

Now we must examine the vanishing sequence at the 1 level in the chains. For both pairs the ramification sequence is $(0,1)$, so the vanishing sequence is $(0,2)$. In particular if $a=2$ then the two pairs are exchanged, and if $a \neq 2$ then the element $\pi_{2, a}$ acts as the identity.

Let us see what the element $\pi_{2,2}$ does to Young tableaux. Referring back to Example 1, the exchange on the corresponding Young tableaux is shown below.

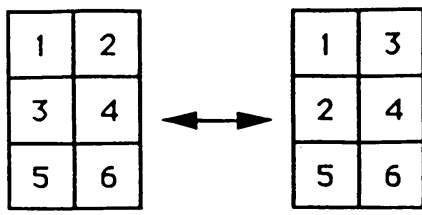

and

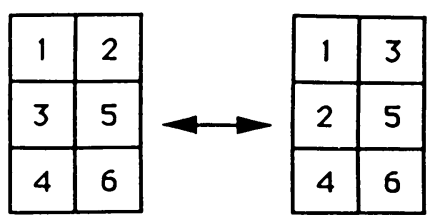


Both pairs are identical except that the 2 and the 3 are exchanged.

Define the distance between two tableaux entries $c=T_{i, j}$ and $d=T_{k, l}$ by $(|k-i|+|l-j|)$ (the so-called taxi-cab distance).

Motivated by Example 2, we have the following proposition.

Proposition 1. The generator $\pi_{c, a}$ acts by exchanging $c$ and $c+1$ in all Young tableaux in which $c$ and $c+1$ lie in different rows and columns and are separated by distance $a$.

It is at this point that the error in [EH] becomes relevant. Because the value of $a$ was originally written as the difference in the ramification sequences, Bercov and Proctor [BP] interpreted (through no fault of their own) the element $\pi_{c, a}$ as exchanging $c$ and $c+1$ when they are separated by $a$ columns.

Proof. Given two chains of Schubert cycles with indices $\underline{\alpha}$ and $\underline{\beta}$ that are exchanged by the monodromy element $\pi_{c, a}$, denote their corresponding Young tableaux by $S$ and $T$. If $S_{i, j}=d$ then

$$
\alpha_{j}^{(d-1)}=\alpha_{j}^{(d)}=d-i .
$$

Likewise, if $d \neq c$ or $d \neq c+1$, then we also have

$$
\beta_{j}^{(d-1)}=\beta_{j}^{(d)}=d-i .
$$

In particular, the two Young tableaux differ only in the entries of $c$ and $c+$ 1. Since $S$ and $T$ are tableaux of distinct integers, $c$ and $c+1$ must be interchanged in the two tableaux. It remains to show that the distance between $c$ and $c+1$ is $a$, and that they lie in different rows and columns.

The two chains $\underline{\alpha}$ and $\underline{\beta}$ differ only in the $j$ th and $k$ th entries at the $c$ th level, so we must have

$$
\begin{aligned}
& \alpha_{j}^{(c)}=\alpha_{j}^{(c-1)}, \\
& \alpha_{k}^{(c)}=\alpha_{k}^{(c-1)}+1, \\
& \beta_{j}^{(c)}=\beta_{j}^{(c-1)}+1, \\
& \beta_{k}^{(c)}=\beta_{k}^{(c-1)},
\end{aligned}
$$

and all other entries the same. For some $i$ and $l$, we have $S_{i, j}=c, S_{l, k}=c+1$ and $T_{l, k}=c, T_{i, j}=c+1$. By definition,

$$
\alpha_{j-1}^{(c-1)}=\alpha_{j-1}^{(c)}=c-i
$$


and

Thus,

$$
\alpha_{k-1}^{(c)}=\alpha_{k-1}^{(c+1)}=c+1-l
$$

$$
a=a_{j-1}^{(c-1)}-a_{k-1}^{(c-1)}=(c-i+j)+(c-l+k)=(l-i)+(j-k),
$$

which is the distance between $c$ and $c+1$ in the tableau $T$. (We are assuming, without loss of generality, that $j>k$.) In particular, $j \neq k$ so $c$ and $c+1$ lie in different columns. If $c$ and $c+1$ were in the same row, then $i=l$ so $\alpha_{j-1}^{(c)}=c-i$ and $\alpha_{k-1}^{(c)}=c-i+1$. However $j>k$, so $\alpha_{j-1}^{(c)} \geq \alpha_{k-1}^{(c)}$ because $\underline{\alpha}^{(c)}$ is a nondecreasing sequence.

\section{Proof of two-transitivity}

Given the above discussion, it suffices to prove that the action described on Young tableaux is two-transitive on $m \times n$ Young tableaux. Since the action is symmetric with respect to rows and columns, we can assume $m<n$. Denote the set of $m \times n$ Young tableaux by $X(m, n)$. Let $H(m, n)$ be the group generated by the elements $\pi_{c, a}$.

Lemma. $H(m, n)$ acts two-transitively on $X(m, n)$.

Proof. Following the lead of Bercov and Proctor [BP], we order the elements of $X(m, n)$ by reading the entries of a tableau down the columns and from left to right, and then ordering the $m n$ entries lexicographically. Denote the minimal tableau by $A$ and the maximal tableau by $Z$. The tableaux $A$ has entries $A_{i, j}=(j-1) m+i$, and $Z$ has entries $Z_{i, j}=(i-1) n+j$.

Let $H(m, n)$ be the group generated by the elements $\pi_{c, a}$. We claim that since $H(m, n)$ is transitive [EH], it suffices to show that given $T$ such that $A<T<Z$, there exists a $\pi$ in $H(m, n)$ such that $\pi(T)<T$ and $\pi(Z)=Z$. Given a pair of tableaux $(T, U)$, by transitivity of $H(m, n)$, we can move it to a pair $(W, Z)$. In turn, this pair can be moved to a pair $\left(W_{1}, Z\right)$ with $W_{1}<W$. Repeating this process we can eventually move our original pair to the pair $(A, Z)$.

Let $T_{p, q}=M+1$ be the first entry (in the lexicographic ordering) that differs from the entries in the minimal tableau. Our strategy is to show that we can replace $M+1$ by $M$ while fixing $Z$. A little thought shows that $M$ lies above and to the right of $M+1$. (The reader is encouraged to make a diagram.) If $T_{r, s}=M$, then $r<p$ and $s>q$. Let $a=(p-r)+(s-q)$, then the element $\pi_{M, a}$ exchanges $M$ and $M+1$. We must check, however, that $\pi_{M, a}$ fixes $Z$. The only consecutive pairs in $Z$ that lie in different rows and columns are the pairs $(n, n+1),(2 n, 2 n+1), \ldots,(m(n-1), m(n-1)+1)$. In each pair, the numbers are a distance of $n$ units apart. Thus, if $a \neq n$ or if $M$ is not divisible by $n$, we are done.

We only have to deal with the case where $n \mid M$ and $a=n$. The proof breaks up into several cases depending on the location of $M-1$. Let $T_{u, v}=M-1$ and note that $u<p$ and $v \geq q$. 
(1) $r<u<p$ and $q<v<s(M-1$ lies strictly between $M$ and $M+1)$. In this case exchanging $M-1$ and $M$ followed by exchanging $M+1$ and $M$ decreases the lexicographic order while fixing $Z$, since after the first exchange, $M$ and $M+1$ is not a distance of $n$ units apart.

(2) $u<r$ and $v>s(M-1$ lies above and to the right of $M)$. The same process as in case (1) works.

(3) $v=q(M-1$ lies in the same column as $M+1)$. In this case, $M-1$ would be forced to lie directly above $M+1$. By assumption, $M-1$ is an entry in the minimal tableau. Thus, any entry in the $q+i$ th column is at least $M+i$. Now, $m<n$, so $M+1$ and $M$ are separated by at least two columns (i.e., $s>p+1)$ since the distance between them is $n$. This is a contradiction.

(4) $u=r . M-1$ is in the same row as $M$, and thus must be adjacent to $M$ (so $v=s-1$ ). This is the hardest case. Cosserve that if $r>1$, or if $M$; then $T_{r, 1} \neq M-s+1$ (i.e., the first $s$ entries of the $r$ th row are not consecutive). Let $c$ be the smallest positive integer such that $T_{r, s-c} \neq M-c$. Since $T_{r, s-1}=$ $M-1$, we have $c \geq 2$. Also, $c \leq s-1<n$. $M-c$ can lie in one of three positions, strictly between $M-c+1$ and $M+1$, above and to the right of $M$, or directly above $M+1$. In this case we can perform the following sequence of exchanges: $(M-c, M-c+1),(M-c+1, M-c+2), \ldots,(M-2, M-1)$. The effect of these exchanges is to move $M-1$ to the original location of $M-c$. We are thus reduced to one of cases (1), (2), or (3). The only thing we have to check is that $Z$ is fixed. This is easy because $M-k$ is not divisible by $n$ when $k<n$ (remember $n \mid M)$.

(4a) $r=1$ and $M=n$. In this case the first row is $1,2, \ldots, n=M$. Here, we proceed by induction. The bottom $m-1$ rows of $T$ form an $(m-1) \times n$ tableau. Let $Y(m, n)$ be the set of Young tableaux with first row $1,2, \ldots, n$. $Y(m, n)$ can be identified with $X(m-1, n)$, and the induced inclusion of $X(m-1, n)$ into $X(m, n)$ preserves the order on $X(m-1, n)$. Let $I(m, n)$ be the subgroup of $H(m, n)$ generated by elements of the form $\pi_{c, a}$ with $c>n . I(m, n)$ acts on $Y(m, n)$. Furthermore, this action is equivalent to the action of $H(m-1, n)$ on $X(m-1, n)$. (An element $\pi_{c, a}$ in $H(m-1, n)$ corresponds to the element $\pi_{c+n, a}$ in $I(m, n)$.) By induction, we can assume that $H(m-1, n)$ acts two-transitively on $X(m-1, n)$. Thus we can decrease the order of $T \in Y(m, n)$ while fixing the maximal element of $Y(m, n)$, which is $Z$.

(5) $v=s . M-1$ is in the same column as $M$, and therefore, must be directly above $M$ (so $u=r-1$ ). Let $c$ be the smallest positive integer, less than $r$, such that $T_{r-c, s} \neq M-c$. If there is no such number, let $c=r$. Since $T_{r-1, s}=M-1$, we have $c \geq 2$. Also $c \leq m$. Looking at the tableau, we see that $M-c$ can lie in one of four possible positions, strictly between $M$ and $M+1$, directly to the left of $M$, directly above $M+1$, or if $c<r$, above and to the right of $M-c+1$. By moving the whole column as in case (4), we can move $M-1$ to the original spot of $M-c$, thus reducing to cases (1), (2), (3), 
or (4). We do not have to worry about moving $Z$ since $M-k$ is not divisible by $n$ when $k<m$ (recall $m<n$ ).

This completes the proof of two-transitivity.

If $m=n$, then the transpose of an $m \times n$ tableau is still an $m \times n$ tableau. Since our action is symmetric with respect to rows and columns, it commutes with the transpose operation. Thus a pair of tableaux $\left(T, T^{t}\right)$ is always moved to a pair $\left(S, S^{t}\right)$ by $H(m, m)$. Therefore, $H(m, m)$ is not two-transitive.

\section{Proof OF THEOREMS 1 AND 2}

We now use the aforementioned theorem of Bochert to conclude the proof of Theorem 1 [BP, Proposition 1].

Theorem (Bochert). Let $G$ be a transitive subgroup of $S_{N}$. If there is an element of $G$ that moves fewer than $N / 3-2 \sqrt{N} / 3$ elements, then $G$ is either the symmetric or alternating group.

In our case $N=N(m, n)$ is the number of $m \times n$ Young tableaux. This number is just [BP],

$$
N(n, m)=(n m) ! \prod_{i=0}^{i=m-1} \frac{i !}{(n+i) !}
$$

If $m \geq 3$ and $n \geq 4$, then the ratio $(N / 3-2 \sqrt{N} / 3) / N>1 / 4$. In order to apply Bochert's theorem, it suffices to prove the following lemma.

Lemma 1. If $m \geq 3$ and $n \geq 4$ then $\pi_{m+n-2, m+n-2}$ moves at most $1 / 4$ of the tableaux.

Proof. Two entries in a tableau are $m+n-2$ units apart if and only if they lie in opposite corners. In particular, $m+n-2$ must lie in the lower left corner and $m+n-1$ lies in the upper right corner, or vice versa. The numbers $1, \ldots, m+n-1$ fill the first row and column. Thus we can identify the elements lying to the right of the first column and below the first row, as a subtableau of size $(n-1) \times(m-1)$. Let $S(m, n)$ be the set of Young tableaux on which $\pi_{m+n-2, m+n-2}$ acts nontrivially.

If $m \geq 4$ we can construct 4 tableaux of size $m \times n$ out of each tableau $T$ in $S(m, n)$. The first, $T_{1}$, is the tableau itself. The second, $T_{2}$, is the tableau where we exchange $m+n$ and $m+n-1$ and leave all other entries the same. The third, $T_{3}$, is the tableau where we exchange $m+n$ and $m+n-2$. The fourth, $T_{4}$, is the tableau constructed by exchanging the pairs $(m+n-2, m+n)$ and $(m+n-1, m+n+1)$.

Given $T, T^{\prime} \in S(m, n)$, we must show that $T_{1}, \ldots, T_{4}$ are distinct from any of the $T_{1}^{\prime}, \ldots, T_{4}^{\prime}$. If $T$ and $T^{\prime}$ differ in any entries other than those of $m+n-2, m+n-1, m+n$, and $m+n+1$, then the $T_{i}$ 's and the $T_{i}^{\prime}$ 's also differ in those entries. Therefore, we may assume that $T$ and $T^{\prime}$ differ only in 


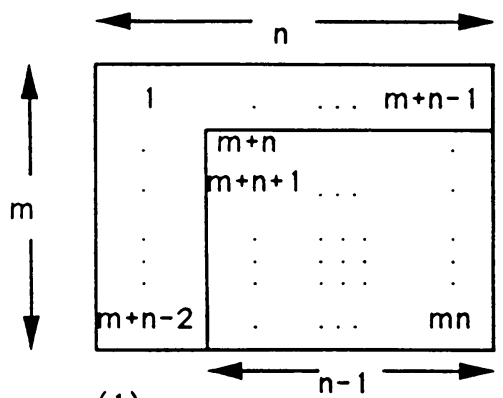

$T^{(1)}$

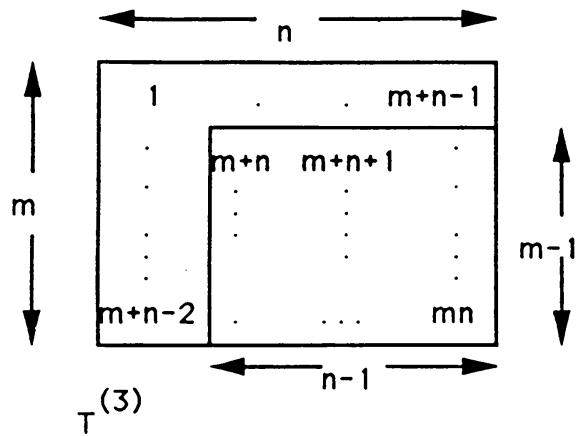

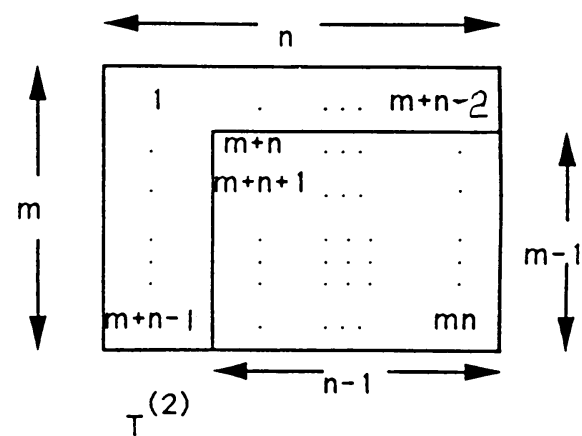

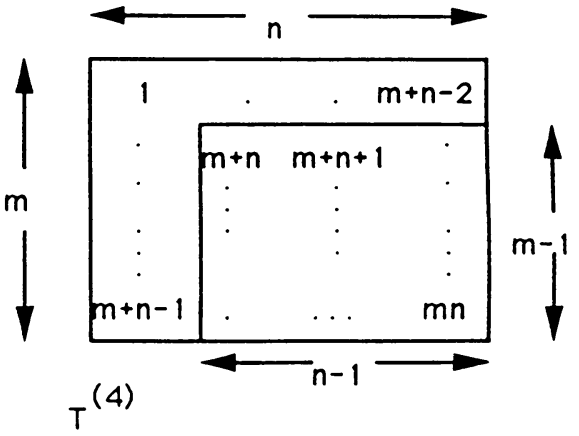

the entries of $m+n-2, m+n-1, m+n$, and $m+n+1$. For tableaux in $S(m, n)$, there are 4 possible configurations for the locations of these entries.

The reader may easily check that the $T_{j}^{(i)}$ 's are all distinct for $i=1, \ldots, 4$.

If $m=3$, then a similar argument shows that for $m$ sufficiently large (even 7 or 8$)$ there are at least 4 elements of $H(m, n)$ for each element of $S(m, n)$. For the remaining cases, direct computation, using the formula for $N(n, m)$ given above, shows that Bochert's bound is satisfied as long as $n \geq 4$.

(The actual ratio is $\frac{|S(n, m)|}{|H(n, m)|}=2\left(\begin{array}{c}n+m-4 \\ m-2\end{array}\right) \frac{N(n-1, m-1)}{N(n, m)}$. If $n=3$ and $m=4$, this ratio is already $5 / 77$. Another possible way to prove the lemma would be to argue that the ratio is monotonically decreasing in $n$ and $m$.)

If $m \geq 3$ and $n \geq 4$, then the permutation $\pi_{m+n-2, m+n-2}$ satisfies the hypothesis of Bochert's theorem. We can therefore conclude that $H(m, n)$ is at least $A_{N}$. This proves Theorem 1 .

If $m=2$ (or $n=2$ ), then it is easy to see (cf. [EH]) that $\pi_{m+n-2, m+n-2}$ is a transposition, proving that $H(2, n)=H(n, 2)=S_{N}(H(2, n)$ is doubly transitive).

To prove the last statement in Theorem 2 , we show that every generator $\pi_{c, a}$ is even if $m=n$ and $n>2$.

Proposition 2. If $m \geq 3$ then every element, $\pi_{c, a}$, of $H(m, m)$ is a product of an even number of disjoint transpositions.

Proof. From the description of $\pi_{c, a}$ above, it is clear that $\pi_{c, a}$ is a product 
of disjoint transpositions. We show that the number of tableaux on which $\pi_{c, a}$ acts nontrivially is divisible by 4 .

Let $G$ be the group generated by a fixed $\pi_{c, a}$ and the transpose operation. As was stated above, $\pi_{c, a}$ commutes with transposition. Also, $\left(\pi_{c, a}\right)^{2}=\mathrm{id}$, and for any $m \times m$ tableau $T,\left(T^{t}\right)^{t}=T$. Therefore, $G$ is a group of order 4. Let $P(m, m)$ be the set of tableaux on which this $\pi_{c, a}$ acts nontrivially. We prove below that $G$ acts freely on $P(m, m)$. Therefore, the number of elements in $P(m, m)$ is divisible by 4 .

Given $T \in P(m, m)$, if $T_{i, j} \notin\{c, c+1\}$, then $\pi_{c, a}(T)_{i, j}=T_{i, j}$, while $T_{i, j}^{t}=T_{j, i}$. Thus $\pi_{c, a}(T) \neq T^{t}$. Also, $\pi_{c, a}(T) \neq T$ and $T^{t} \neq T$. Therefore, the $G$ orbit of $T$ has at least 3 distinct elements. Since $G$ has order 4 , the orbit must have order exactly 4 , implying that $G$ acts freely on $P(m, m)$.

This concludes the proof of the proposition.

If $m \neq n$ we have not, in general, been able to determine the parity of generators in $H(m, n)$.

\section{ACKNOWLEDGMENT}

I would like to thank Vic Reiner and Eric Babson for helpful combinatorial discussions and Joe Harris for suggesting the problem.

\section{REFERENCES}

[ACGH] E. Arbarello, M. Cornalba, P. Griffiths, and J. Harris, The geometry of algebraic curves, Springer-Verlag, New York, 1984.

[BP] R. Bercov and R. Proctor, Solution of a combinatorially formulated monodromy problem of Eisenbud and Harris, Ann. Sci. École Norm. Sup. 20 (1987), 241-250.

[EH] D. Eisenbud and J. Harris, Irreducibility and monodromy of certain families of linear series, Ann. Sci. École Norm. Sup. 20 (1987), 65-87.

Department of Mathematics, Massachusetts Institute of Technology, Cambridge, MASSACHUSETTS 02139 